\title{
A systematic review and narrative synthesis of group self-management interventions for adults with epilepsy
}

\author{
Amelia Smith ${ }^{1}$, Alison McKinlay², Gabriella Wojewodka ${ }^{2}$ and Leone Ridsdale $2^{2^{*}}$
}

\begin{abstract}
Background: Epilepsy is a serious and costly long-term condition that negatively affects quality of life, especially if seizures persist on medication. Studies show that people with epilepsy (PWE) want to learn more about the condition and some educational self-management courses have been trialled internationally. The objectives of this review were to evaluate research and summarise results on group self-management interventions for PWE.

Methods: We searched Medline and PsycINFO for results published in English between 1995 and 2015. Only studies evaluating face-to-face, group interventions for adults with epilepsy were included. Heterogeneity in study outcomes prevented the carrying out of a meta-analysis; however, a Cochrane style review was undertaken.

Results: We found eleven studies, nine of which were randomised controlled trials. There were variable standards of methodological reporting with some risk of bias. Seven of the studies used quality of life as an outcome, with four finding statistically significant improvements in mean total score. Two found an improvement in outcome subscales. One study included some additional semi-qualitative data.

Conclusions: We identified promising trends in the trials reviewed. In particular, there were significant improvements in quality of life scales and seizure frequency in many of the interventions. However, considerable heterogeneity of interventions and outcomes made comparison between the studies difficult. Courses that included psychological interventions and others that had a high number of sessions showed more effect than short educational courses. Furthermore, the evidence was predominantly from pilot studies with small sample sizes and short follow-up duration. Further research is needed to better evaluate the role of group self-management interventions in outpatient epilepsy management.
\end{abstract}

Keywords: Self-management education, Epilepsy, Patient-education, Quality of life

\section{Background}

Epilepsy is a long-term condition characterised by recurrent seizures, with a prevalence of around $1 \%$ in the general population [1,2]. Common consequences of living with epilepsy include driving limitations, detrimental effects on education, unemployment, and diminished psychological wellbeing [3]. Stigma, frequency of seizures, and healthcare experiences also affect quality of life (QoL) in people with epilepsy (PWE) [4].

\footnotetext{
* Correspondence: leone.ridsdale@kcl.ac.uk

${ }^{2}$ Institute of Psychiatry, Psychology \& Neuroscience, Academic Neuroscience

Centre, King's College London, PO Box 57, London SE5 8AF, UK

Full list of author information is available at the end of the article
}

Epilepsy has significant financial and social costs. Direct costs are associated with a high rate of emergency admission that occurs with poorly-controlled epilepsy [5]. Emergency service use makes up the majority of admissions for epilepsy. Among all long-term conditions, epilepsy is the sixth most common cause of emergency admission in the United Kingdom [6, 7]. Reducing unnecessary emergency admissions is a key factor in helping to relieve financial pressure on healthcare services. Another major social issue is the indirect cost of epilepsy due to lost employment [8]. The health and social costs could be reduced and QoL improved via better outpatient management. However, around $40 \%$ of those diagnosed have poorly-controlled epilepsy and 
continue to have two or more seizures per year, [3] despite using antiepileptic drugs (AEDs). These figures highlight missed opportunities for epilepsy self-management.

Management of long-term conditions requires selfefficacy and empowerment, enabling patients to live as independently as possible and reducing the need to go to hospital [9]. For other long-term conditions, strategies for enabling such behaviour have been attempted within self-management education courses. A diabetes group intervention used in the United Kingdom, called DESMOND, is a cost-effective intervention, shown to improve biopsychosocial outcomes [10-12]. The programme is structured and can be run over one to two days for six hours in total [12]. Content is based on social learning theory [13] and is integrated into standard outpatient care for diabetes.

Early research evidence from North America [14] and Germany [15] suggested that group self-management courses had the potential to have a positive effect on health outcomes in PWE. The interest in group selfmanagement for PWE has grown; however, the evidence base for developing self-management groups as standard outpatient care for PWE is still small. The objectives of this review were to evaluate recent research and summarise results of group self-management interventions for PWE. This was undertaken in the context that our group was conducting a trial of group self-management education intervention in the UK [16].

\section{Methods}

\section{Study eligibility criteria \\ Population}

PWE, adults aged 16 or over, without learning disabilities (due to the markedly different approaches required for educational programs in these populations [17]).

\section{Intervention}

Group self-management interventions were the focus of this review, irrespective of the study objectives (i.e., education, behavioural therapy, or a combination were included). We were interested in the psychological and social elements of face-to-face, group self-management courses. Studies using telemedicine were therefore excluded, as they are not provided face-to-face in groups.

\section{Comparison}

Treatment as usual or waitlist control.

\section{Outcomes of interest}

There was particular interest in QoL as this is the outcome favoured by the National Institute for Health and Care Excellence (NICE) $[18,19]$. However, as there is no fixed consensus on the best measure for evaluating group interventions, we also included studies assessing other outcomes such as seizure frequency, psychological state, self-efficacy, and knowledge of epilepsy.

\section{Exclusion criteria}

Studies reporting trial protocol without results, one-toone interventions, web- or telephone-based interventions, and samples including people with learning disabilities, children or non-epileptic seizures.

We searched for papers published from 1990 to 2015. A randomised controlled trial is considered the "gold standard" research design for evaluating the efficacy of an intervention; [20] however, we extended search criteria to include other forms of clinical trial (i.e., controlled outcomes design).

\section{Search strategy}

We conducted electronic searches of the databases Medline and PsycINFO using the following keywords: epilepsy, seizures, self-care/self-efficacy, patient education/education programme, self-management, group intervention/complex intervention. A manual search of reference lists was performed to identify further relevant studies. For specific strategies for database searches refer to Appendix 1.

\section{Quality appraisal}

All studies were assessed for quality using the CONSORT guidelines for reporting clinical trials [21]. Studies were assigned a number between 0 (description absent) and 2 (satisfactory description) according to how fully they described the four sections: trial design (methodology), participants (study sample and characteristics), interventions and study outcomes. Appraisal was carried out by two independent assessors and studies were discussed as a group to resolve any disagreements. The Cochrane approach was used to categorise the studies into 'high,' 'low' or 'unclear' risk of bias with regard to random sequence generation and allocation concealment [22]. As it is not possible to double-blind a group self-management intervention, this was not included in the quality assessment.

\section{Results \\ Study selection}

The first author conducted the initial literature search, which was repeated by the second author. The initial Medline search resulted in 42 papers being identified (Fig. 1). After examining the titles and abstracts, nine were unrelated and were excluded. Five studies on nonepileptic seizures (psychogenic seizures and febrile convulsions) were excluded also [23-27]. Five more were excluded as their participant population consisted of children (under 16) or patients with learning disabilities. Of the remaining papers, six were identified as involving a telephone or web-based intervention and were also excluded. Finally, some studies were excluded because no 


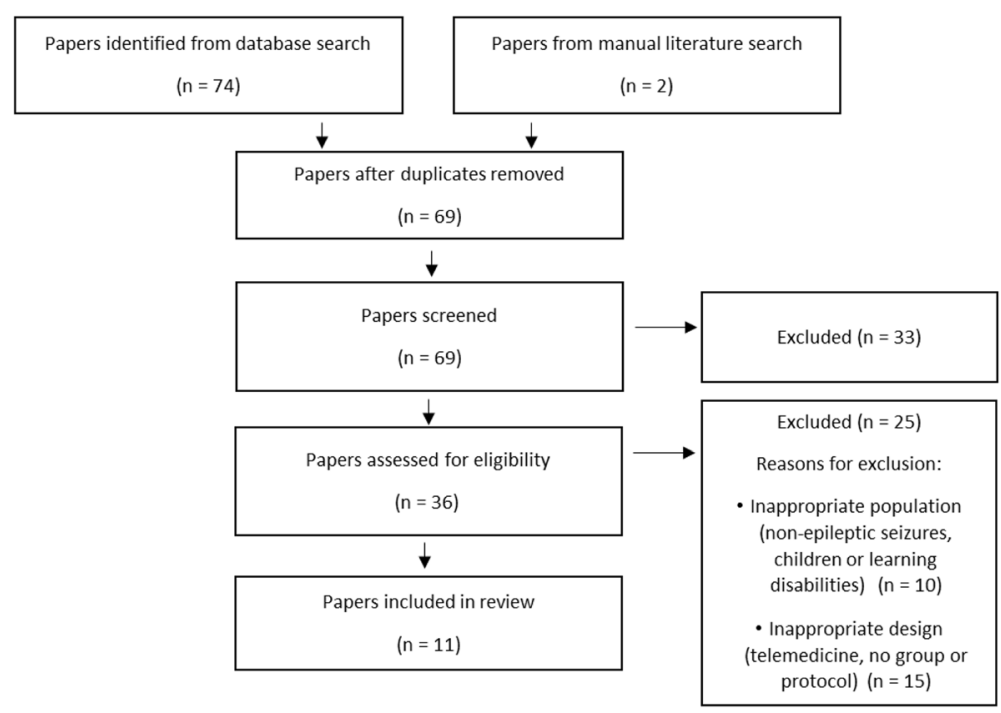

Fig. 1 Search tree

group intervention was administered. The papers described either individual care programmes or trial protocols with no results.

PsycINFO returned 32 results; three new papers were identified, but none were eligible for inclusion (two protocols, one telemedicine). Two studies were included from the manual literature search, resulting in 11 studies in total.

\section{Search results}

Of the 11 studies included with this review (Table 1), nine were described by their authors as randomised controlled trials $[15,28-30,31,32-35]$ and the remaining two as controlled outcome designs [36, 37]. The first study included a matching strategy for allocating participants to control or treatment group [37]. The second study used random assignment but did not provide more specific protocol information [36]. After synthesising review results, we established that due to outcome heterogeneity a meta-analysis would not be possible.

All studies focused on the effects of a group intervention in PWE, with one exclusively recruiting women [28] and another looking at older adults [32]. Most studies targeted poorly-controlled epilepsy; however, the definition of 'poorly-controlled' varied and four studies did not specify a minimum seizure frequency.

We assessed 11 studies for quality and risk of bias (Table 2). For most studies, the participants and outcome measures were well described. Trial design was the least well described throughout the 11 studies followed by the intervention description. Eight of the 11 studies had low risk of bias for the generation of the sequence allocating participants to treatment groups. In most study designs for self-management courses, the participants and facilitators will be un-blinded. Other research staff, such as those assessing participants and analysing data should have remained blinded to minimise bias. The concealment of group allocation was not described in the majority of studies.

\section{Facilitators and treatment groups}

Interventions were categorised as educational, $[15,28,29$, $33,34]$ psychological (e.g. behavioural therapy) [30, 32, 37] and psychoeducational programmes (i.e. both) [31, 35]. Psychological and psychoeducational interventions were delivered by psychologists, whereas educational sessions were delivered by a range of practitioners. In two studies, $[34,36]$ instructors' qualifications were not clearly specified (e.g. 'researcher' or 'staff'). In one study, [15] facilitators were specified in a previous publication to be physicians, nurses, psychologists, social workers and occupational therapists.

The treatment of the control group varied across the studies. In six of the studies, [15, 28, 29, 31, 37, 33] controls received treatment as usual (TAU). Additional input was given to the controls alongside TAU in one study [33] in the form of two short telephone calls. In two, [30, 32] a control for attention was offered, for example, supportive therapy, involving equivalent attention from professionals, but without any active teaching or advice. Three studies [34-36] used a waitlist control design and offered intervention materials after follow-up.

\section{Outcomes}

The most frequently assessed outcome measure was QoL, used in seven studies included within this review [15, 28-30, 31, 35, 37]. The Quality of Life in Epilepsy questionnaire (QOLIE-31/QOLIE-89) [38, 39] was 


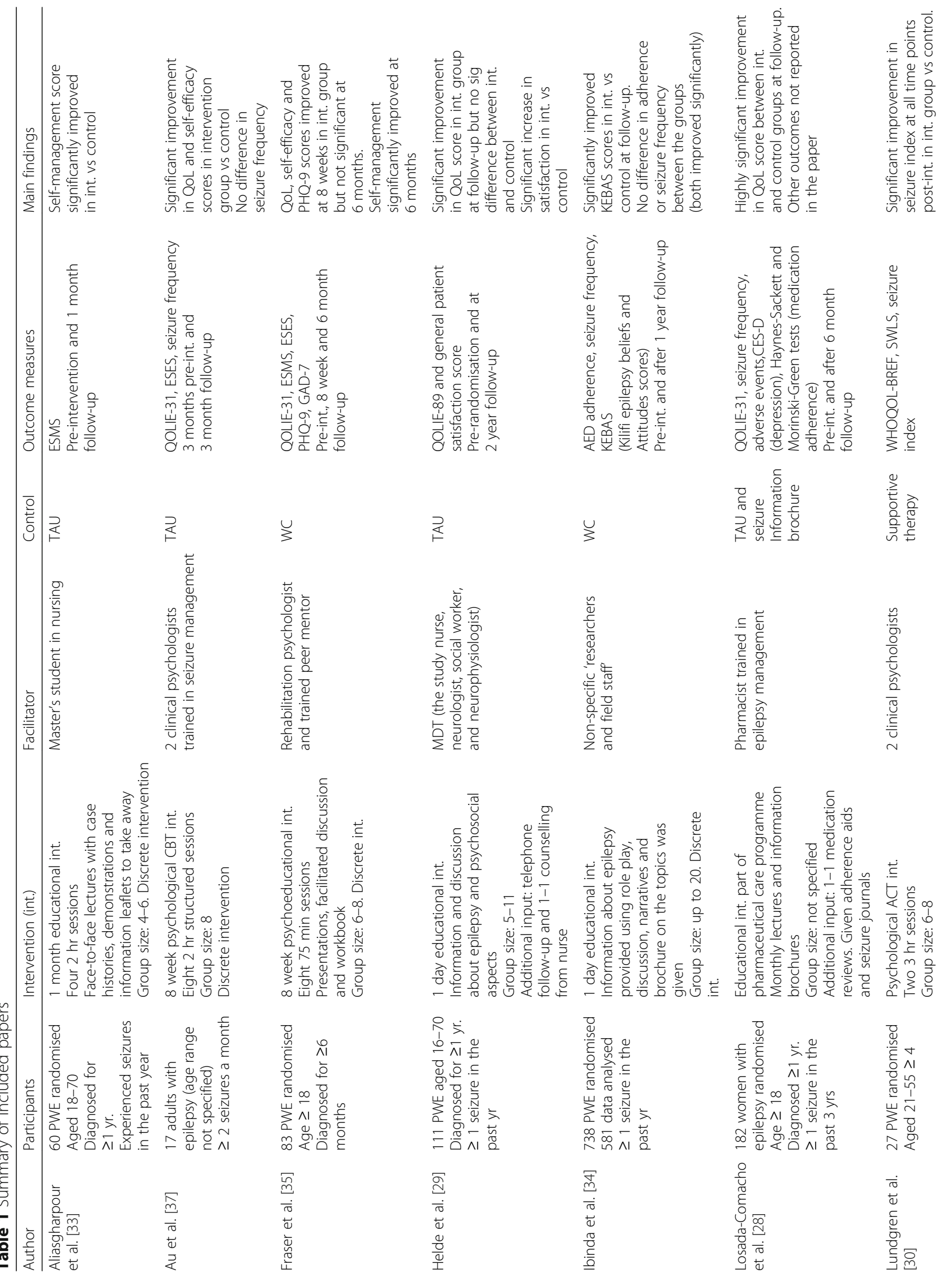




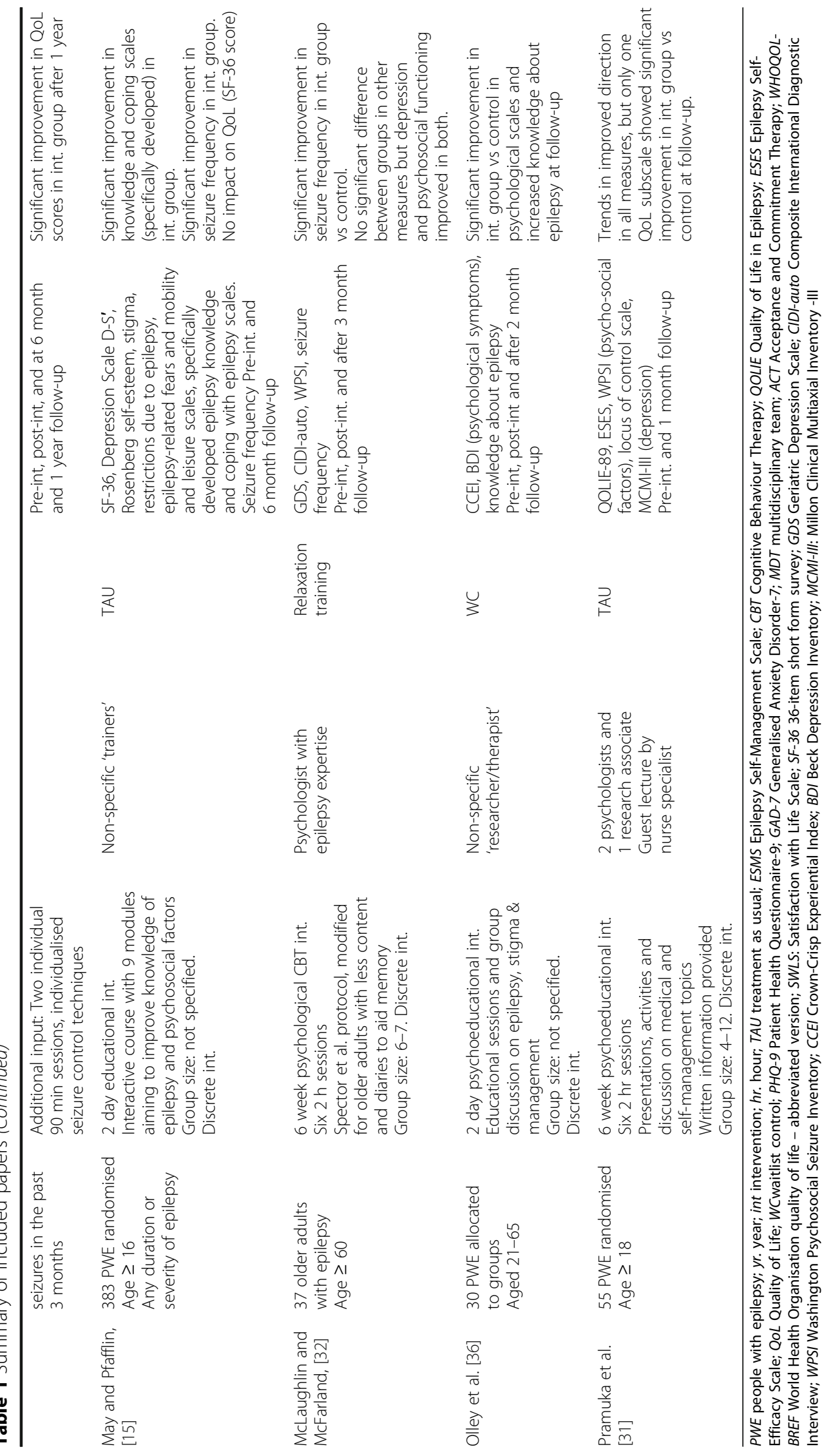


Smith et al. BMC Neurology (2017) 17:114

Page 6 of 10

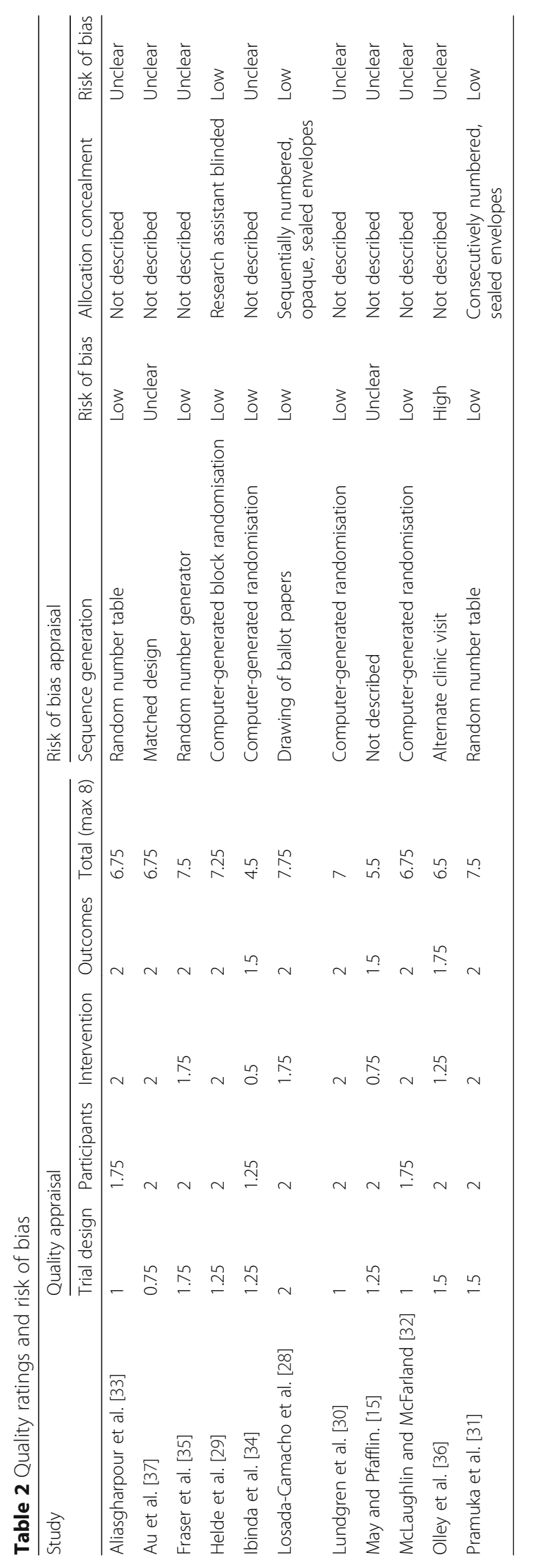


commonly chosen to assess it [28, 29, 31, 35, 37]. Six studies $[15,28,30,32,34,37]$ measured seizure frequency as a marker of control and three assessed self-efficacy, [31, 35, 37] using the Epilepsy SelfEfficacy Scale (ESES) [40]. Two studies [33, 35] used a specific self-management outcome measure called the Epilepsy Self-Management Scale (ESMS) [41]. Different measures were used to assess psychological symptoms, such as the Patient Health Questionnaire (PHQ-9) [42]. Comparison between the studies was complicated, due to the heterogeneity of outcome measures that were used.

\section{Follow-up}

The duration of follow-up ranged from one month [33] to two years [29] with a median follow-up period of six months. All studies recorded outcome measures at baseline before the intervention and at the end of follow-up. Four also took further measures immediately post-intervention or halfway through the follow-up period $[30,32,35,36]$.

\section{Overview of findings Quality of life}

Of the seven studies using QoL outcome measures, three showed no significant difference in total QoL scores between the intervention and control groups $[15,29,31]$. Fraser et al. showed a significant improvement in QOLIE-31 scores eight weeks post-intervention, but no statistical significance between means after six months [35].

Two psychological interventions showed an improvement in QoL scores at follow-up. The cognitive behavioural therapy study demonstrated a significant QOLIE-31 score improvement in the intervention group compared with the control at the end of a three month follow-up [37]. Emotional Wellbeing was the only subscale that was significantly increased. In the trial by Lundgren et al [30]. there was a significant improvement in WHOQOL-BREF score (World Health Organisation Quality of Life Assessment abbreviated version) [43] in the acceptance and commitment therapy group, compared to supportive therapy at the final one year follow-up. The Satisfaction with Life Scale (SWLS) [44] score was improved at all three time points after the intervention.

One education-focused study showed an improvement in QoL score. The trial by Losada-Camacho et al. found significant improvement in QOLIE-31 after six months [28]. The intervention package was offered to women only, and focused on pharmaceutical aspects of epilepsy self-management. As one of the co-interventions, participants received advice and monitoring at set intervals from a pharmacist. At six-month follow-up, the total score increased in the intervention group by 12.45 points compared with a 2.61 increase in the controls.

\section{Seizure frequency}

Six studies measured seizure frequency. Three found that seizure frequency decreased significantly in the intervention group compared with controls [28, 30, 32]. However, two studies did not find a significant difference in seizure frequency post-intervention. Au et al. described a small improvement in the intervention group but this was not significant, [37] whereas, Ibinda et al. found a decrease in both groups but no difference between them [34]. One study did not report seizure frequency despite collecting this data [28]. The reason for this was unclear; however, it may be due to poor compliance with seizure diaries as only $37.5 \%$ of participants returned them.

\section{Self-management and self-efficacy}

Two studies using the ESMS showed promising findings after group intervention delivery. Aliasgharpour et al. found a significant difference between the groups postintervention with an increase in self-management score in the intervention group but none in the control [33]. Likewise, Fraser et al. found a treatment effect favouring the intervention at eight weeks which, although small, remained significant at the six month follow-up [35].

Three studies used the ESES to measure self-efficacy $[31,35,37]$. Fraser et al. found a significant treatment effect in ESES score after eight weeks but this did not persist at six months. Au et al. found a significant improvement in score in the intervention group compared to control. Findings from Pramuka et al. showed a trend towards improvement in the intervention group but this was not significant.

\section{Psychological symptoms}

Two of the six studies which used symptom scales indicating psychological comorbidity showed statistically significant improvement $[35,36]$. Fraser et al. found a statistical significant change in PHQ-9 [39] score with reductions in depressive symptom severity at eight weeks postintervention; however, the difference between intervention and control was not statistically significant at six months. Olley et al. found an improvement in psychological state compared with controls but, with only two months follow-up, no long-term benefit was demonstrated [36]. The others found improvements which were not significant $[28,31,32]$. In the study by Losada-Camacho et al. the psychological outcome results were not reported, [28] nor were they published elsewhere.

\section{Discussion}

Quality assessment revealed gaps in the methodological reporting of many studies, especially regarding trial design. Risk of bias was assessed and most papers fell into the 'unclear' category due to insufficient description of treatment concealment. We paid particular attention to who delivered 
each intervention and how thoroughly this was described. Information about the role and training of facilitators is needed in order to replicate an intervention and apply it to other healthcare settings.

The usefulness of study findings is somewhat diminished in those that lacked thorough reporting of treatment fidelity. Most interventions were delivered multiple times, sometimes by different facilitators; however, only one study discussed means of ensuring treatment fidelity but the results were not presented [30]. Group interventions may not be delivered consistently and without reporting on measures to prevent deviation, it is uncertain whether interventions were delivered as planned.

All studies reported significant improvement in the intervention group compared with controls in at least one measure. More than half of the studies assessing QoL found some positive effect from the intervention but the duration of that effect is varied. Five studies repeated the QoL measures at least six months after the intervention and only two found a sustained significant improvement $[28,30]$. These both consisted of sessions which were spread out over time, which may be a better intervention strategy than one-off courses. Fraser et al. also delivered their intervention over a period of 8 weeks but were unable to demonstrate a significant effect at 6 month follow-up [35]. They suggested the addition of a booster course to the programme. It is likely that other factors may also influence the duration of improvement.

It was difficult to identify mechanisms that make a group intervention for PWE effective. This is particularly true of co-interventions, where group effects cannot be separated out from additional support. Psychological interventions performed well on QoL and seizure frequency (Table 1) [36, 37, 32] although they had very small sample sizes ( $n=17,27$ and 37$)$. This suggests that including behavioural therapy in self-management interventions may be important to affect QoL.

Although some studies found positive trends for QoL improvements from baseline measurements, the scores were not necessarily statistically different between study groups. Many were pilot studies with small sample sizes and may not have had adequate power to detect important changes. One study that encountered this problem calculated a required sample size of 180 participants but recruitment difficulties resulted in a final sample of only 55 [31]. Larger-scale trials are needed to explore this further.

Another factor that may have contributed to the lack of significant effect is the use of unsuitable outcomes. Two studies found improvements only in measures developed specifically for the trial, despite including a variety of other outcomes $[15,34]$. This suggests that the existing scales may not be optimal for evaluating effects of self-management interventions in this setting.
The diversity of outcome measures used across all studies made comparison difficult. There were five scales used to assess QoL, of which two were epilepsy-specific. Four studies did not use any QoL questionnaires although their chosen outcomes (e.g. seizure frequency) can be assumed to affect QoL. This highlights lack of consensus on the most appropriate measures for complex interventions in epilepsy.

Only one study used semi-qualitative data in their evaluation, collecting written comments from participants in an open-ended satisfaction survey [35]. They received positive comments about the intervention with $47 \%$ of respondents mentioning the value of meeting other PWE. However, response rates were uncertain and there were no interviews to explore participants' views. Guidelines from the Medical Research Council recommend the inclusion of qualitative methods as part of a process evaluation for complex interventions [45]. In light of this, it would be useful to collect further qualitative data on how and why group self-management interventions are beneficial.

The diversity of study settings also complicates interpretation. As trials were conducted across 13 countries with wide-ranging cultural and socioeconomic backgrounds, baseline standards of care and health literacy were likely different. Furthermore, participants in each setting may not be representative of the wider population. This was particularly relevant in the Seattle-based study which typically recruited highly educated participants, some of whom were volunteers, limiting the generalisability of their findings to other western countries in which health care is provided to all socio-economic groups [35].

There are several limitations associated with the present review. We were unable to perform a meta-analysis due to significant heterogeneity of outcome measures observed across the studies. Moreover, not all Cochrane review tools were utilised when evaluating the quality of studies included. Findings from the review may also be limited due to biases arising from initial search criteria (i.e., article language and publication date).

\section{Suggestions for future research}

Based on the evidence available to date, it seems that QoL is rarely affected long term by educational interventions. Thus to have a better chance of affecting QoL, self-management interventions should include psychological components. If group self-management education is to be offered as part of standard outpatient care for PWE, as DESMOND is for people with diabetes, then future research should examine the feasibility and costeffectiveness of implementation. Although this review did not specifically search for published articles with health economics data, we found no discussion of the cost of implementing self-management education courses for PWE in the community. This would be valuable in the 
context of similar programmes being implemented in countries with public healthcare systems.

\section{Conclusions}

The studies evaluating group self-management interventions for PWE found encouraging results. There is some evidence that psychoeducational measures can be delivered to improve self-management, seizure control, and QoL in adults with poorly-controlled epilepsy. The MOSES programme has been offered in German-speaking countries for over a decade and other countries are investigating similar interventions [15]. Promising findings, along with demand from patients for more information about epilepsy, are indications for continued interest in group self-management interventions for PWE.

This review illustrates the need for clarity regarding outcome measures in this field of epilepsy research. Additionally, large-scale trials of group self-management interventions, combining quantitative, qualitative, and cost-effectiveness data, are required in the future.

\section{Appendix 1}

Search strategies

\section{Medline (Ovid) search strategy}

1. exp. Epilepsy/

2. epilep*

3. seizures

4. exp. Self Care/

5. exp. Self Efficacy/

6. exp. Patient Education as Topic/

7. educational program

8. self-management

9. group intervention

10. complex intervention

11. 1 or 2 or 3

12. Limit 11 to (English language and yr. = "1995 Current")

13. 4 or 5 or 6 or 7 or 8 or 9 or 10

14. Limit 13 to (English language and yr. = "1995 Current")

\subsection{2 and 14}

16. Limit 15 to (clinical trial or controlled clinical trial or pragmatic clinical trial or randomized controlled trial)

\section{PsycINFO search strategy}
1. Exp Epilepsy/
2. epilep*
3. seizures
4. 1 or 2 or 3
5. self-management

6. exp. Self Efficacy/

7. group intervention

8. complex intervention

9. patient education

10. self care

11. educational program*

12.5 or 6 or 7 or 8 or 9 or 10 or 11

13. 12 and 4

14. limit 13 to (English language and yr. = "1995Current")

\begin{abstract}
Abbreviations
DESMOND: Diabetes education and self-management for ongoing and newly diagnosed; ESES: Epilepsy self-efficacy scale; ESMS: Epilepsy self-management scale; MOSES: Modular service package epilepsy; PHQ-9: Patient health questionnaire 9; PWE: People with epilepsy; QoL: Quality of life; QOLIE: Quality of life in epilepsy; SWLS: Satisfaction with life scale; TAU: Treatment as usual; WHOQOL-BREF: World health organisation quality of life abbreviated version
\end{abstract}

\section{Acknowledgements}

The authors would like to acknowledge our clinical trial administrator, Carly Pearson.

\section{Funding}

This research was in part funded by the National Institute for Health Research (NIHR), under the Health Technology Assessment Programme (09/165/01), including salaries for AM and GW. There are no further funding sources to declare. The views and opinions expressed by authors in this publication are those of the authors and do not necessarily reflect those of the NHS, NIHR, MRC, CCF, NETSCC, HTA programme, or Department of Health.

Availability of data and materials

Not applicable as no datasets were generated.

Authors' contributions

AS was responsible for writing the first major draft of the manuscript, evaluating each article included with the review, and carrying out subsequent revisions of the manuscript. AM evaluated each of the articles included with the review and carried out moderate revisions of the manuscript in preparation for publication. GW evaluated each paper included with the review, contributed to the development of the manuscript and provided feedback throughout manuscript preparation. LR conceived of the study and its design and methods, and provided supervision and feedback throughout. All authors read and approved the final manuscript.

\section{Competing interests}

There are no further competing interests to declare.

Consent for publication

Not applicable.

Ethics approval and consent to participate Not applicable.

\section{Publisher's Note}

Springer Nature remains neutral with regard to jurisdictional claims in published maps and institutional affiliations.

\section{Author details}

${ }^{1}$ GKT School of Medicine, King's College London, London SE1 1UL, UK. ${ }^{2}$ Institute of Psychiatry, Psychology \& Neuroscience, Academic Neuroscience Centre, King's College London, PO Box 57, London SE5 8AF, UK. 
Received: 13 March 2017 Accepted: 6 June 2017

Published online: 17 June 2017

\section{References}

1. Longmore M, Wilkinson I, Baldwin A, Wallin E. Oxford handbook of clinical Medicine. 9th ed. Oxford: Oxford University Press; 2014.

2. Smithson WH, Walker MC, editors. ABC of epilepsy. 1st ed. Chichester: WileyBlackwell; 2012.

3. Moran NF, Poole K, Bell G, Solomon J, Kendall S, McCarthy M, et al. Epilepsy in the United Kingdom: seizure frequency and severity, anti-epileptic drug utilization and impact on life in 1652 people with epilepsy. Seizure. 2004; 13(6):425-33.

4. Jacoby A, Snape D, Baker GA. Determinants of quality of life in people with epilepsy. Neurol Clin. 2009;27(4):843-63.

5. Bruce M, Griffiths C, Brock A, Majeed A. Trends in mortality and hospital admissions associated with epilepsy in England and Wales during the 1990s. Health Stat Q. 2004 Spring;21:23-9.

6. Noble AJ, Goldstein LH, Seed P, Glucksman E, Ridsdale L. Characteristics of people with epilepsy who attend emergency departments: prospective study of metropolitanhospital attendees. Epilepsia. 2012;53(10):1820-8.

7. Health and Social Care Information Centre ( $\mathrm{HSCIC}$ ), Clinical Indicators team. CCG Outcomes Indicator Set: Emergency Admissions. 2013; Available at: http://www.hscic.gov.uk/catalogue/PUB10584/ccg-ind-toi-mar-13-v4.pdf. Accessed October 28, 2015.

8. Cockerell OC, Hart YM, Sander JW, Shorvon SD. The cost of epilepsy in the United Kingdom: an estimation based on the results of two populationbased studies. Epilepsy Res. 1994;18(3):249-60.

9. NHS England. Domain 2: Enhancing Quality Of Life For People With LongTerm Conditions. Available at: http://www.england.nhs.uk/resources/ resources-for-ccgs/out-frwrk/dom-2/\#help. Accessed 16 December 2015.

10. Carey ME, Mandalia PK, Daly H, Gray LJ, Hale R, Martin Stacey L, et al. Increasing capacity to deliver diabetes self-management education: results of the DESMOND lay educator non-randomized controlled equivalence trial. Diabetic Med. 2014;31(11):1431-8.

11. Gillett M, Dallosso HM, Dixon S, Brennan A, Carey ME, Campbell MJ. Delivering the diabetes education and self management for ongoing and newly diagnosed (DESMOND) programme for people with newly diagnosed type 2 diabetes: cost effectiveness analysis. BMJ. 2010;341

12. Davies MJ, Heller S, Skinner TC, Campbell MJ, Carey ME, Cradock S, et al. Effectiveness of the diabetes education and self management for ongoing and newly diagnosed (DESMOND) programme for people with newly diagnosed type 2 diabetes: cluster randomised controlled trial. BMJ. 2008; 336(7642):491-5

13. Bandura A. Self-efficacy: toward a unifying theory of behavioral change. Psychol Rev. 1977;84(2):191-215.

14. Helgeson DC, Mittan R, Tan SY, Chayasirisobhon S. Sepulveda epilepsy education: the efficacy of a psychoeducational treatment program in treating medical and psychosocial aspects of epilepsy. Epilepsia. 1990;31(1):75-82

15. May TW, Pfäfflin M. The efficacy of an educational treatment program for patients with epilepsy (MOSES): results of a controlled. Randomized Study Epilepsia. 2002;43(5):539-49.

16. Kralj-Hans I, Goldstein LH, Noble AJ, Landau S, Magill N, McCrone P. Selfmanagement education for adults with poorly controlled epILEpsy (SMILE (UK)): a randomised controlled trial protocol. BMC Neurol. 2014:14

17. Durand M-A, Gates B, Parkes G, Zia A, Friedli K, Barton G, et al. Wordless intervention for epilepsy in learning disabilities (WIELD): study protocol for a randomized controlled feasibility trial. Trials. 2014;15(1):455.

18. National Institute for Health Care and Excellence. Guide to the methods of technology appraisal 2013. 2013; Available at: http://nice.org.uk/process/ pmg9 accessed 4 March 2017.

19. Chidgey J, Leng G, Lacey T. Implementing NICE guidance. J R Soc Med. 2007;100(10):448-52

20. Sibbald B, Roland M. Understanding controlled trials. Why are randomised controlled trials important?. BMJ: British Medical Journal. 1998;316(7126):201.

21. Schulz KF, Altman DG, Moher D, CONSORT Group. CONSORT 2010 Statement: updated guidelines for reporting parallel group randomised trials. BMJ. 2010;340:c332.

22. Higgins J, Altman D, Sterne J. Chapter 8: assessing risk of bias in included studies. 2011; Available at: http://handbook.cochrane.org/chapter_8/8_ assessing_risk_of_bias_in_included_studies.htm. Accessed 13 December 2015.
23. Chen D, Maheshwari A, Franks R, Trolley G, Robinson J, Hrachovy R. Brief group psychoeducation for psychogenic nonepileptic seizures: a neurologist-initiated program in an epilepsy center. Epilepsia. 2014;55(1):156-66.

24. Paul $F$, Jones MC, Hendry C, Adair PM. The quality of written information for parents regarding the management of a febrile convulsion: a randomized controlled trial. J Clin Nurs. 2007;16(12):2308-22.

25. Huang MC, Liu CC, Huang CC. Effects of an educational program on parents with febrile convulsive children. Pediatr Neurol. 1998;18(2):150-5.

26. Riaz H, Comish S, Lawton L, Scheepers B. Non-epileptic attack disorder and clinical outcome: a pilot study. Seizure. 1998;7(5):365-8.

27. Thompson N, Connelly L, Peltzer J, Nowack WJ, Hamera E, Hunter EE. Psychogenic Nonepileptic seizures: a pilot study of a brief educational intervention. Perspectives in Psychiatric Care. 2013 Apr;49(2):78-83.

28. Losada-Camacho M, Guerrero-Pabon MF, Garcia-Delgado P, MartínezMartinez F. Impact of a pharmaceutical care programme on health-related quality of life among women with epilepsy: a randomised controlled trial (IPHIWWE study). Health Qual Life Outcomes. 2014;12:162.

29. Helde G, Bovim G, Bråthen G, Brodtkorb E. A structured, nurse-led intervention program improves quality of life in patients with epilepsy: A randomized, controlled trial. Epilepsy \& Behavior 2005 11;7(3):451-457.

30. Lundgren T, Dahl J, Melin L, Kies B. Evaluation of acceptance and commitment therapy for drug refractory epilepsy: a randomized controlled trial in South Africa - a pilot study. Epilepsia. 2006;47(12):2173-9.

31. Pramuka M, Hendrickson R, Zinski A, Van Cott AC. A psychosocial selfmanagement program for epilepsy: a randomized pilot study in adults. Epilepsy Behav. 2007;11(4):533-45.

32. McLaughlin DP, McFarland K. A randomized trial of a group based cognitive behavior therapy program for older adults with epilepsy: the impact on seizure frequency, depression and psychosocial well-being. Journal of Behaviour Medicine. 2011;34(3):201-7.

33. Aliasgharpour $M$, Dehgahn Nayeri $N$, Yadegary MA, Haghani $H$. Effects of an educational program on self-management in patients with epilepsy. Seizure. 2013;22(1):48-52

34. Ibinda F, Mbuba CK, Kariuki SM, Chengo E, Ngugi AK, Odhiambo R, et al. Evaluation of Kilifi epilepsy education Programme: a randomized controlled trial. Epilepsia. 2014;55(2):344-52.

35. Fraser R, Johnson E, Lashley S, Barber J, Chaytor N, Miller J, et al. PACES in epilepsy: results of a self-management randomized controlled trial. Epilepsia. 2015;56(8):1264-74.

36. Olley BO, Osinowo HO, Brieger WR. Psycho-educational therapy among Nigerian adult patients with epilepsy: a controlled outcome study. Patient Educ Couns. 2001:42(1):25-33.

37. Au A, Chan F, Li K, Leung P, Li P, Chan J. Cognitive-behavioral group treatment program for adults with epilepsy in Hong Kong. Epilepsy Behav. 2003;4(4):441-6.

38. Cramer JA, Perrine K, Devinsky O, Bryant-Comstock L, Meador K, Hermann B. Development and cross-cultural translations of a 31-item quality of life in epilepsy Inventory. Epilepsia. 1998;39(1):81-8.

39. Devinsky O, Vickrey BG, Cramer J, Perrine K, Hermann B, Meador K, et al. Development of the quality of life in epilepsy Inventory. Epilepsia. 1995; 36(11):1089-104

40. Dilorio C, Faherty B, Manteuffel B. The development and testing of an instrument to measure self-efficacy in individuals with epilepsy. J Neurosci Nurs. 1992;24(1):9-13.

41. Dilorio C, Shafer PO, Letz R, Henry TR, Schomer DL, Yeager K. Behavioral, social, and affective factors associated with self-efficacy for self-management among people with epilepsy. Epilepsy Behav. 2006;9(1):158-63.

42. Kroenke K, Spitzer RL, Williams JB. The PHQ-9: validity of a brief depression severity measure. J Gen Intern Med. 2001:16(9):606-13.

43. WHOQOL Group. Development of the World Health Organization WHOOOL-BREF quality of life assessment. Psychol Med. 1998:28(03):551-8.

44. Diener E, Emmons RA, Larsen RJ, Griffin S. The satisfaction with life scale. J Pers Assess. 1985;49(1):71-5.

45. Craig P, Dieppe P, Macintyre S, Michie S, Nazareth I, Petticrew M. Developing and evaluating complex interventions: the new Medical Research Council guidance. Int J Nurs Stud. 2013;50(5):587-92. 